\title{
Prevalência de estratégias para cessação do uso do tabaco na Atenção Primária à Saúde: uma revisão integrativa
}

\author{
The prevalence of strategies for cessation of tobacco use \\ in primary health care: an integrative review
}

Meire de Deus Vieira Santos ${ }^{1}$

Stella Vieira Santos ${ }^{1}$

Maria do Carmo Gullaci Guimarães Caccia-Bava ${ }^{1}$

${ }^{1}$ Faculdade de Medicina de Ribeirão Preto, Universidade de São Paulo. Av. Bandeirantes 3900 , Monte Alegre. 14048-900 Ribeirão Preto SP Brasil. meirevsantos@hotmail.com

\begin{abstract}
The habit of tobacco use/smoking, which is a major concern of Primary Health Care (PHC), is a serious public health problem and the main avoidable cause of death in the world. The relevance of actions, whose focus is to facilitate the cessation of this habit, motivates the discussion of studies that have different approaches to tackle this issue by seeking to train PHC professionals accordingly. A search was conducted in the Lilacs, MEDLINE and Web of Science databases for recent scientific publications (2010-2015). The key words were combined with Boolean operators and, after analysis of the articles found, 75 are discussed in this article since they have strategies with a higher prevalence in PHC. The conclusion drawn is that the brief or intense individual approach using the 5A method (Transtheoretical Model) is the most widely adopted, as well as bupropion and nicotine replacement patches. The increasing use of hard technology requires new studies that examine their impact on the treatment of smokers. It was clearly revealed that there is a need for health professionals to be better prepared to address the issue with the users, in addition to a lack of stimulus and proper conditions to work in the PHC team directly reflecting scientific advances in clinical practice.
\end{abstract}

Key words Smoking, Tobacco use cessation, Tobacco use cessation products, Primary Health Care
Resumo O hábito de fumar, ou tabagismo, preocupação da Atenção Primária à Saúde (APS), é um grave problema de saúde pública e a principal causa de morte evitável no mundo. A relevância de ações, cujo foco seja facilitar a cessação deste vício, motiva a discussão de estudos que apresentam diferentes abordagens para tal enfrentamento visando contribuir para a formação dos profissionais da APS. Utilizou-se as bases de dados Lilacs, Medline e Web of Science considerando as produções científicas recentes (2010 a 2015). Os descritores foram combinados a operadores boleanos e, após análise dos artigos encontrados, 75 são discutidos nesta revisão por apresentarem estratégias de maior prevalência na APS. Conclui-se que a abordagem individual breve ou intensa a partir do método dos 5A's (Modelo Transteórico) é a mais adotada, assim como os fármacos adesivos de Nicotina e Bupropiona. O uso crescente de tecnologia dura necessita de novos estudos que averiguem os seus impactos no tratamento a tabagistas. Evidenciou-se a necessidade de o profissional de saúde ser mais bem preparado para abordar o tema com os usuários, além de carecer do estímulo e das condições próprias para atuar na equipe de APS refletindo diretamente os avanços científicos em sua prática clínica.

Palavras-chave Hábito de fumar, Abandono do uso de tabaco, Produtos para o abandono do uso de tabaco, Atenção primária à saúde 


\section{Introdução}

O Programa Saúde da Família (PSF) possui importante função na organização das práticas na Atenção Primária à Saúde (APS) devido a sua concepção em múltiplas dimensões, desde a prevenção a doenças até a recuperação, a promoção e a reabilitação em saúde. Atualmente desdobrou-se na Estratégia de Saúde da Família (ESF) visando garantir atendimento integral e cuidado continuado a partir da articulação entre a atenção básica e os demais pontos de complexidade da rede de saúde ${ }^{1}$.

No entanto, muitos fatores podem prejudicar as ações na APS aumentando a morbimortalidade, dentre os quais o tabagismo ou hábito de fumar, doença crônica derivada da dependência de Nicotina e que acarreta problemas de saúde passíveis de se tornarem de extrema gravidade ${ }^{1}$.

A efetividade das intervenções de promoção da saúde junto a adultos tabagistas que recebem cuidados primários de saúde, com base em modelos teóricos de mudança de comportamento para modificar os principais fatores de estilo de vida (atividade física, dieta, álcool e tabaco), tem sido alvo de estudos realizados entre 2002 e 2012 que apontam a ausência de artigos tratando explicitamente das estratégias de intervenção e teorias de mudança de comportamento ${ }^{2}$.

O preparo do profissional de saúde que aborda o tabagista também tem sido questionado, pois na maioria das vezes mostrou-se insuficiente para localizar com sucesso o conhecimento existente para tal fim, de modo que políticas públicas são essenciais para aprimorar a formação destes profissionais consolidando os serviços de $\mathrm{APS}^{3}$.

De acordo com a literatura, os profissionais da saúde têm o tabaco como fator de risco para inúmeras doenças como câncer, asma, Doença Pulmonar Obstrutiva Crônica (DPOC), infecções respiratórias e doenças cardiovasculares. A Organização Mundial da Saúde (OMS) o considera como problema de saúde pública por ser a principal causa de morte evitável no mundo, tendo sua prevalência reduzida gradativamente em algumas regiões e grupos populacionais menos vulneráveis ${ }^{1,4,5}$.

A prevalência mundial de tabagismo foi de 1,3 bilhão no ano de 2013, considerando indivíduos maiores de 15 anos. Deu-se $70 \%$ em países em desenvolvimento onde 19,2\% correspondiam ao sexo feminino. Estima-se que no Brasil vivam 20,1 milhões de fumantes, $14,8 \%$ da população maior de 18 anos $^{4,6}$.
O perfil dos fumantes brasileiros no Programa Nacional de Controle de Tabaco (PNCT) indica a média de idade de 49,32 anos +/- 11,82 anos, $71 \%$ mulheres, sendo que cerca de metade dos pacientes parou de fumar. Níveis altos de $\mathrm{Ni}$ cotina associaram-se a níveis baixos de cessação do tabagismo, havendo diminuição na probabilidade de permanecerem fumadores em todo o primeiro mês de tratamento. Após três semanas de tratamento apenas 19\% não parou de fumar, a probabilidade diminuindo em $2 \%$ por ano adicional de idade ${ }^{7}$.

A cessação do tabagismo mostra-se salutar em qualquer momento da vida, produzindo benefícios consideráveis para a saúde pública ${ }^{8}$. O custo-benefício do tratamento da pessoa tabagista se mostra favorável nos cuidados em saúde, sobretudo quando relacionado às doenças crônicas. Assim, uma intervenção clínica contínua pode levar a reduções significativas na prevalência do tabagismo e na taxa de visitas ao consultório para tratar doenças associadas?.

No Brasil, os usuários encontram dificuldades na obtenção de bons resultados no abandono ao vício junto ao Sistema Único de Saúde (SUS), por meio da participação no Plano Nacional de Controle do Tabagismo (PNCT). O acesso ao programa, assim como o controle da ansiedade, a estruturação e organização dos grupos de apoio e uma boa atuação dos profissionais de saúde no controle do hábito, são pontos importantes a serem aprimorados ${ }^{10}$.

Com o objetivo, pois, de identificar os conhecimentos produzidos relacionados aos estudos científicos sobre a cessação do tabagismo, identificando novos métodos e estratégias que auxiliem o profissional de saúde, subsidiando sua abordagem aos usuários da APS que buscam estímulos para a cessação desta dependência, esta revisão integrativa de literatura propõe abarcar artigos produzidos recentemente considerando os anos de 2010 e 2015.

O recorte temático e a descrição dos estudos que compreendem a abordagem individual breve ou intensa a partir do método dos 5A's (base da Entrevista Motivacional), a prescrição de adesivos de Nicotina e Bupropiona, a necessária formação continuada dos profissionais de saúde para a abordagem aos pacientas tabagistas e uma melhor compreensão sobre o impacto do uso de tecnologia dura para o acompanhamento neste tipo de tratamento mostraram-se como estratégias prevalecentes nas práticas de APS para cessação do uso do tabaco e sobre elas discorremos a seguir. 


\section{Método}

Visando ampliar o espectro de informações sobre o problema-alvo, a partir de busca nas bases de dados Literatura Latino-Americana e do Caribe em Ciências da Saúde (LILACS), Medical Literature Analysis and Retrieval Sistem on-line (MEDLINE) e Web of Science buscou-se por pesquisas experimentais ou não associadas a informações teóricas e empíricas.

Utilizou-se os Descritores em Ciências da Saúde (DeCS) combinados com operadores booleanos: "Hábito de fumar" OR "Abandono do uso do tabaco" OR "Produtos para o abandono do uso de tabaco" AND "Atenção Primária à Saúde" e seus respectivos termos em língua inglesa. Considerou-se como critérios de inclusão: i) artigos disponíveis integralmente em português, inglês ou espanhol e ii) periódicos nacionais e internacionais indexados nos últimos cinco anos, compreendendo de 2010 a 2015, visando a abordagem de estudos recentes. Excluíram-se artigos repetidos entre as bases.

Foram selecionados 323 artigos (39 indexados na Lilacs, 203 na Medline e 81 na Web of Science) submetidos à análise dos resumos para verificação da coincidência entre tema e foco do estudo. A identificação dos métodos usados pelos pacientes que romperam sua dependência do cigarro e/ou as estratégias com as quais os profissionais de saúde abordaram os usuários favorecendo tal rompimento, assim como a manutenção da abstinência, foram integrados nesta análise caracterizando os artigos que apresentavam subsídios efetivos à resolutividade da cessação do hábito, os principais elencados neste trabalho devido à limitação de espaço deste texto ${ }^{11,12}$.

Por centrar o seu foco em trabalhos já publicados nas bases públicas de dados anteriormente referidas o presente estudo exime-se de aprovação por Comitê de Ética e não recebeu financiamento de qualquer ordem para a sua realização.

\section{Resultados e discussão}

Após análise, delimitou-se o aprofundamento de 111 artigos pertinentes ao tema "cessação do tabagismo" (10 artigos indexados na Lilacs, 69 na Medline e 32 na Web of Science), conforme Quadro 1.

Realizou-se, então, a análise crítica individual dos textos viabilizando sua classificação em sete categorias temáticas relativas às estratégias prevalecentes na APS para o tratamento ao paciente tabagista. Limitamo-nos a apresentar e discutir estratégias predominantes em mais de $10 \%$ dos estudos, conforme Quadro 2: Abordagem individual (35,51\% de prevalência na APS; 38 artigos publicados); Abordagem ao profissional de saúde (20,56\% de prevalência; 22 artigos); Prescrição de medicamentos (18,69\% de prevalência; 20 artigos) e Acompanhamento e/ou apoio com o uso de tecnologia dura $(10,28 \%$ de prevalência; 11 artigos), totalizando 91 artigos científicos nacionais e internacionais publicados com relação ao tema nos últimos cinco anos $(81,98 \%$ do total de 111 artigos analisados profundamente).

Segundo estudos, a chance de um paciente tabagista receber intervenções para cessação do uso do tabaco varia de acordo com sua condição socioeconômica, sendo as práticas de aconselhamento e/ou farmacoterapia mais frequentes na população de baixa renda. Os fumantes que não receberam intervenção geralmente possuíam ensino superior, estabilidade financeira, matrimônio sem filhos e acesso a jornais e banda larga, de modo que grupos com taxas de intervenção particularmente baixa podem ser mais bem direcio-

Quadro 1. Combinação entre Descritores em Ciências da Saúde (DeCS) e operadores booleanos utilizada nas bases de dados LILACS, Web of Science e MEDLINE em setembro de 2015. Elaborado pela autora, 2015.

\begin{tabular}{|c|c|c|c|}
\hline Base de dados & Descritores +Operadores Booleanos & Artigos Indexados & Incluídos no Estudo \\
\hline $\begin{array}{l}\text { Literatura Latino- } \\
\text { Americana e do Caribe } \\
\text { em Ciências da Saúde } \\
\text { (LILACS) }\end{array}$ & $\begin{array}{l}\text { "Hábito de fumar" OR "Abandono } \\
\text { do uso de tabaco" OR "Produtos para } \\
\text { o abandono do uso de tabaco" AND } \\
\text { "Atenção Primária à Saúde" }\end{array}$ & 39 & $\begin{array}{r}9,0 \% \\
{[10 \text { artigos] }}\end{array}$ \\
\hline $\begin{array}{l}\text { Medical Literature } \\
\text { Analysis and Retrieval } \\
\text { Sistem on-line } \\
\text { (MEDLINE) }\end{array}$ & \multirow[t]{2}{*}{$\begin{array}{l}\text { "Smoking" OR "Abandonment } \\
\text { of tobacco use" OR "Products } \\
\text { abandonment tobacco use" AND } \\
\text { "Primary Health Care" }\end{array}$} & 203 & $\begin{array}{r}62,16 \% \\
\text { [69 artigos] }\end{array}$ \\
\hline Web of Science & & 81 & $\begin{array}{r}28,83 \% \\
\text { [32 artigos] }\end{array}$ \\
\hline Total & & 323 & 111 \\
\hline
\end{tabular}


Quadro 2. Categorização temática individual a partir da análise dos 111 artigos selecionados das bases de dados LILACS, Web of Science e MEDLINE em setembro de 2015 e organizados conforme a prevalência do tipo de estratégia utilizada na APS para a cessação do tabaco. Elaborado pela autora, 2015.

\begin{tabular}{|c|c|c|c|}
\hline \multicolumn{3}{|c|}{ Categorização } & Prevalência \\
\hline \multirow[t]{6}{*}{${ }^{*}$ Abordagem Individual } & \multicolumn{2}{|c|}{ Intervenção breve/aconselhamento face-a-face } & \multirow{6}{*}{$\begin{array}{l}35,51 \% \\
{[38 \text { artigos] }}\end{array}$} \\
\hline & \multicolumn{2}{|c|}{ Intervenção intensiva } & \\
\hline & \multicolumn{2}{|c|}{ Entrevista motivacional } & \\
\hline & \multicolumn{2}{|c|}{ Espirometria para controle de emissão de carbono } & \\
\hline & \multicolumn{2}{|c|}{ Estratégia dos 5as/3as } & \\
\hline & \multicolumn{2}{|c|}{ Terapia cognitivo-comportamental } & \\
\hline \multirow{6}{*}{$\begin{array}{l}{ }^{*} \text { Abordagem ao profissional de } \\
\text { Saúde }\end{array}$} & \multirow{4}{*}{$\begin{array}{l}\text { Educação } \\
\text { para }\end{array}$} & Formação/ conhecimento & \multirow{6}{*}{$\begin{array}{l}20,56 \% \\
{[22 \text { artigos] }}\end{array}$} \\
\hline & & Saber abordar o paciente & \\
\hline & & Registro em prontuário & \\
\hline & & $\begin{array}{l}\text { Inserir tabagismo na rotina } \\
\text { de anamnese }\end{array}$ & \\
\hline & \multirow{2}{*}{$\begin{array}{l}\text { Sensibilização para } \\
\text { Cessar o tabagismo }\end{array}$} & Orientar o paciente & \\
\hline & & & \\
\hline \multirow[t]{3}{*}{${ }^{\star}$ Prescrição de Medicamentos } & \multicolumn{2}{|l|}{ Vareniclina } & \multirow{3}{*}{$\begin{array}{l}18,69 \% \\
{[20 \text { artigos }]}\end{array}$} \\
\hline & \multicolumn{2}{|l|}{ Bupropiona } & \\
\hline & \multicolumn{2}{|l|}{ Nicotina } & \\
\hline${ }^{\star}$ Acompanhamento e/ou apoio & \multicolumn{2}{|c|}{ Uso de tecnologia dura (Tel./ e-mail/) } & $\begin{array}{l}10,28 \\
{[11 \text { artigos }]}\end{array}$ \\
\hline \multirow[t]{4}{*}{ Teóricos } & \multicolumn{2}{|l|}{ Modelo Transteórico } & \multirow{4}{*}{$\begin{array}{l}7,47 \%[8 \\
\text { artigos }]\end{array}$} \\
\hline & \multicolumn{2}{|l|}{ Estratégia dos 5 as/3as } & \\
\hline & \multicolumn{2}{|c|}{ Tabagismo e doenças psiquiátricas } & \\
\hline & \multicolumn{2}{|c|}{ Cultura organizacional (Tempo/financiamento) } & \\
\hline \multirow[t]{4}{*}{ Outros } & \multicolumn{2}{|c|}{ Fumar em público gera vergonha e culpa } & \multirow{4}{*}{$\begin{array}{l}4,67 \% \\
{[5 \text { artigos }]}\end{array}$} \\
\hline & \multicolumn{2}{|c|}{ Estimulação fotoelétrica de pontos da orelha } & \\
\hline & \multicolumn{2}{|c|}{ Incentivos financeiros } & \\
\hline & \multicolumn{2}{|c|}{ Esperança, religião e religiosidade } & \\
\hline \multirow[t]{2}{*}{ Intervenção em grupo } & \multicolumn{2}{|l|}{ Apoio } & $2,8 \%$ \\
\hline & \multicolumn{2}{|l|}{ Educação e saúde } & [3 artigos] \\
\hline
\end{tabular}

Nota: O símbolo * (asterisco) indica categorias temáticas discutidas a partir de 74 artigos no presente trabalho em função de sua prevalência no conjunto de estudos levantados. Os demais temas não estão devidamente descritos em função do enfoque dado pelas autoras diante da limitação de espaço desta produção.

nados por meio da mídia/internet e intervenções baseadas no local de trabalho ${ }^{13}$.

Os profissionais de saúde se mostram fragilizados pessoal e estruturalmente para a condução do processo terapêutico de pacientes usuários de drogas como os tabagistas, o que incluiria práticas de aconselhamento e farmacoterapia que, associadas, possuem taxa de abstinência maior do que a estimada para pacientes tratados com tais práticas isoladas $^{14,15}$.

Verificou-se o predomínio nos estudos dos últimos cinco anos da abordagem individual ao tabagismo (35,51\% dos artigos) com intervenções breves, aplicação de entrevista motivacional, utilização da estratégia dos 5A’s e Terapia cognitivo-comportamental (TCC) com apoio de equi- pe multiprofissional durante consulta de saúde. Destaca-se a realização de espirometria para controle de emissão de carbono visando à sensibilização do paciente com relação à sua função respiratória ${ }^{16-19}$.

A abordagem ao profissional de saúde mostrou-se predominante nos estudos (20,56\%) e foi identificada como necessária no sentido de educação permanente. Apontou-se a necessidade de inserção na rotina do paciente do questionamento sobre o hábito, além do devido registro em prontuário por profissional preparado e não tabagista, sob o risco de dificultar a cessação do hábito de fumar por parte do paciente ${ }^{20-22}$.

Os médicos da APS, o governo e os responsáveis por formular políticas de saúde podem inse- 
rir, pois, equipes bem preparadas e treinadas na linha de frente de seus atendimentos para tornar a prevenção clínica efetiva tanto em nível prático (recursos e qualificação profissional) quanto em nível do sistema de saúde (financiamento para cuidados clínicos preventivos desde a medicação até o apoio de pessoal) $)^{20,23-25}$.

A prescrição de medicamentos ocupou 18,69\% da atenção dos pesquisadores, mostrando-se como estratégia subutilizada e descritas com maior frequência em intervenções clínicas que visam diminuir o uso de tabaco ao invés de promover a sua cessação ${ }^{23}$.

O uso de tecnologia dura por meio da computação e com função de incentivadora da abstinência $(10,28 \%$ das ocorrências nesta revisão integrativa) tem se mostrado cada vez mais como prática de acompanhamento/apoio por meio do envio de SMS, e-mail, além das redes sociais e aplicativos $^{26-28}$.

Estratégias para a cessação do hábito de fumar que versam exclusivamente sobre metodologias disponíveis aos profissionais de saúde - modelos como o Transteórico e a estratégia dos 5 A's (Abordar, Aconselhar, Avaliar, Assistir e Acompanhar) - tornam-se alvo de publicações futuras ${ }^{2,29,30}$, visto que a intervenção para cessação tabágica com base nesses modelos aumenta significativamente a abstinência em dois anos de seguimento entre os fumantes que visitam centros de cuidados primários ${ }^{30-32}$.

O uso de medicamentos subsidiados associados a intervenções e campanhas públicas deve estar previsto nas políticas de combate ao tabagis$\mathrm{mo}^{8,33}$. A intervenção intensiva e individualizada utilizando entrevista, terapias e medicamentos adaptados ao estágio de mudança motivacional do paciente favorece a abstinência e se mostra viável e eficaz na APS, com taxa de cessação de 26,1\% após um ano ${ }^{34-36}$.

Intervenções multicomponentes melhoram os resultados, embora estudos futuros devam ser feitos sobre sua relação custo-eficácia ${ }^{37}$. A implementação da estratégia dos 3 A’s (Averiguar, Animar, Assessorar) propostos pela OMS, bem como a gestão sistemática do indivíduo em risco com a participação ativa do médico e enfermeiro de família durante o processo de atendimento foi um modelo utilizado com sucesso em Cuba para manejo e prevenção do câncer de pulmão ${ }^{38}$.

Nos artigos analisados a intervenção breve com aconselhamento face a face mostrou-se frequente, com tempo limitado e foco na mudança de comportamento do paciente ${ }^{39-41}$. No entanto, apesar de o aconselhamento médico breve ser eficaz na modificação de hábito na maioria dos fumantes, apenas uma pequena proporção permanece abstinente depois de um ano, sendo que conselhos breves durante as consultas de rotina aumentam a taxa de abandono do tabagismo mesmo havendo subnotificação dessas ocorrências $^{16,21}$

Uma única intervenção breve mostrou-se mais eficaz em relação ao tabaco do que em relação ao álcool e ainda mais eficaz se abordados na mesma intervenção ${ }^{42}$. A intervenção individual aplicada nos mesmos moldes que o do programa antitabagismo no SUS, com aconselhamento durante visitas face a face, apresenta cessação tabágica do dobro do grupo não controle em estudo feito com indígenas australianos ${ }^{43}$.

$\mathrm{O}$ envio e recebimento de mensagens por meio de smartphones e e-mail foram amplamente citados, mostrando-se efetivos na intervenção intensiva para cessação do tabagismo, não havendo ainda configuração voltada para a $\mathrm{APS}^{44}$. Com relação a programas na Web, há dificuldade em demonstrar sua eficácia em comparação com cuidados habituais, embora em situações específicas a intervenção por e-mail mostre-se eficiente quando há desejo prévio em abandonar o hábito ${ }^{26-28}$.

A espirometria, exame através do qual se pode estimar a emissão de carbono pelo paciente tabagista, é também apoio extra para a cessação tabágica $^{17}$. CO-oximetria mostrou-se de baixo custo, não invasiva e rápida permitindo abordar os fumantes na APS diante de sua eficácia já atestada como auxiliar no reforço de atividades de cessação do tabagismo,promovendo redução do hábito com maior custo-efetividade naqueles que utilizam estratégias de apoio como mensagens de texto e aconselhamento individual ou em grupo, além de surtir efeitos em intervenção motivacional entre fumantes em condições reais de prática clínica $^{8,17-19}$.

Intervenções intensivas com seguimento de pelo menos seis meses por parte da enfermagem podem ser prejudicadas quando breves ou prestadas por enfermeiros cujo papel principal não é a promoção da saúde ou a cessação do tabagismo diante da não adoção de comportamentos importantes na entrevista motivacional, como perguntas abertas e reflexões complexas ${ }^{45,46}$. Em atendimento exclusivamente ligado ao tabagismo verificou-se que aos tabagistas com dificuldade em abandonar o hábito a intervenção intensiva (10 a 15 minutos) é mais efetiva do que a intervenção breve ( 1 a 5 minutos) em consulta na APS ${ }^{47}$.

Recomenda-se aos médicos de APS que forneçam educação ou aconselhamento breve para 
prevenir a iniciação do tabagismo em crianças e adolescentes em idade escolar sem evidenciar, contudo, o quê deve ser feito para fumantes regulares desta faixa etária na qual não se encontra efetividade em medidas comportamentais e uso de Bupropiona ${ }^{48,49}$.

A farmacoterapia para minimizar sintomas da síndrome de abstinência e facilitar a abordagem ao tabagista deve estar associada ao aconselhamento comportamental e a consultas regulares para ser efetiva ${ }^{50}$, onde os medicamentos descritos no PNCT compreendem a Terapia de Reposição de Nicotina (TRN) por meio de adesivo transdérmico, goma de mascar e pastilhas (nicotínicos), além da Bupropiona (não nicotínico) ${ }^{10}$.

A Vareniclina (não nicotínico), provavelmente a farmacoterapia mais eficaz para cessação do tabagismo, é menos usada que a TRN devido ao seu alto custo. Pacientes aderentes a esse fármaco foram duas vezes mais propensos a ter sucesso em parar de fumar, além de a substância apresentar poucos eventos adversos e ser tolerada por dependentes de opiáceos e/ou pacientes em acompanhamento por outras especialidades, devido ao abuso de substâncias, DPOC, HAS ou depressão. O acompanhamento médico ativo é medida válida para otimizar o tratamento ${ }^{51-57}$.

O apoio na cessação do tabagismo a partir de clínicas especializadas, com tratamento em grupo associado à TRN ou Vareniclina, obteve mais sucesso do que aqueles que receberam tratamento individualizado na APS com aconselhamento e um tipo de terapia farmacológica ${ }^{58}$.

As taxas de abstinência para tratamento de tabagismo (62,1\% Vareniclina, 56,9\% Bupropiona e 52,3\% TRN aos 6 meses de tratamento e $57,4 \%, 52,9 \%$ e $47,1 \%$ aos 12 meses, respectivamente) evidenciam a Vareniclina como alternativa adequada em comparação com a Bupropiona e a TRN, podendo desempenhar papel na prevenção de recaídas ${ }^{59,60}$.

Com relação aos obstáculos para a cessação do tabagismo, estudo qualitativo verificou que profissionais de saúde não fumantes consideram o tabagismo um tema importante a ser discutido com seus pacientes, embora não possam consistentemente abordá-lo durante as consultas clínicas devido às limitações dos sistemas de saúde, aos equívocos e mal-entendidos, às barreiras socioculturais e limitações pessoais, o que os profissionais afirmam prejudicar a mudança no estilo de vida dos pacientes ${ }^{60-62}$.

Oportunidades de triagem e aconselhamento breve para cessação do uso do tabaco foram em grande parte perdidos pelos médicos, de modo que se sugeriu a incorporação do status de tabagista nos sinais vitais clínicos com o potencial de melhorar taxas de triagem de uso de tabaco e subsequente cessação $0^{63,64}$.

O fato de o próprio profissional de saúde ser tabagista é obstáculo encontrado pelos usuários, sendo necessária a abordagem a esses profissionais para melhoria na qualidade da assistência em APS ${ }^{25,65,66}$. A compreensão sobre as barreiras para deixar de fumar experimentada pelos profissionais de saúde é necessária para elaborar programas de cessação culturalmente relevantes e multidimensionais, que abordem a normalização vigente do tabagismo na família, no trabalho e na comunidade ${ }^{20,25}$.

Espera-se que o médico participe ativamente da discussão de cessação de tabagismo, expectativa não correspondida segundo trabalho realizado em Unidade de Saúde da Família em Cuiabá, onde as orientações sobre tabagismo, atividade física e dieta não foram valorizadas nas consultas ou não foram devidamente anotadas no prontuário do paciente, o que se deve à dificuldade para abordar o tabagismo em seus atendimentos, referindo desconhecimento ou inabilidade até mesmo em países desenvolvidos indicando a necessidade do planejamento da capacitação desses profissionais ${ }^{22,67,68}$.

Finalmente, a incorporação de diferentes formas de expressão durante a Entrevista Motivacional ajuda a construir relacionamentos de cuidados de saúde centrados no doente, oferecendothe oportunidade para refletir sobre o consumo de tabaco e favorecendo indivíduos com baixa motivação para a mudança. Ainda, os dados obtidos podem ser incluídos na formação profissional visando melhorar a qualidade da abordagem, além do registro eletrônico de saúde aprimorar o feedback sobre cada atendimento gerando informações em saúde ${ }^{24,69-73}$.

Além de médicos e enfermeiros, estudantes de curso superior da área da saúde podem executar atividades de promoção da saúde e prevenção de danos e agravos relacionados ao tabaco visando o público de escolas, a capacitação de Agentes Comunitários de Saúde e a divulgação do Dia Mundial Sem Tabaco (31 de maio) participando, ainda, de programas de rádios para a sensibilização da clientela de cessação tabágica, o que impacta positivamente a APS ao aumentar o sucesso na melhoria das oportunidades de outras ações preventivas ${ }^{16,74}$. 


\section{Considerações finais}

A literatura consultada permitiu identificar que as orientações para redução de danos ligados ao uso do tabaco envolvem aconselhamento e sensibilização sobre os problemas vinculados ao hábito de fumar, o fornecimento de produtos licenciados que contém Nicotina, assim como não nicotínicos de uso em curto ou longo prazo, o fornecimento de materiais de autoajuda, apoio comportamental, educação e formação de profissionais capacitados para tal serviço.

Apontou, ainda, que o acesso a programas eficazes de cessação do tabagismo é fundamental para redução de morbimortalidade relacionada ao tabaco, devendo as parcerias ser pensadas de maneira intersetorial, assim como o uso de tecnologia dura merece estudos mais aprofundados diante do potencial de atuarem como elementos de apoio para o público mais jovem.

Se por um lado, o custo dos medicamentos disponíveis para cessação do tabagismo (goma de Nicotina, adesivo de Nicotina, Bupropiona e Vareniclina) é mais elevado do que o preço de uma embalagem de cigarros, por outro isto indica a necessidade de países que possuem serviços de apoio à cessação do tabaco prestar cuidados em nível de sociedade com menores custos e de forma mais acessível. Do mesmo modo, os currículos de graduação de profissionais de saúde devem incluir formação suficiente sobre os males e estudos atuais relativos ao tabagismo, além da modificação de estilo de vida, pois os profissionais atuantes na APS carecem da educação médica continuada.

O tabagismo vigente entre os profissionais da área da saúde tem demonstrado ser um limitador das intervenções tanto para a cessação quanto para o controle do tabaco, devendo antes o profissional ser abordado para que ele possa, posteriormente, abordar o paciente.
O entendimento de resiliência pelos profissionais de APS detém potenciais lições para a promoção da saúde e apoio à prevenção do consumo de tabaco na cessação por grupos de risco ${ }^{69}$, ajudando a superar a falta de recursos humanos e treinamento específico considerando o desenvolvimento local, a participação e aceitação das mudanças pela população, além do esforço para o desenvolvimento profissional e apoio dentro de uma estrutura de segurança cultural ${ }^{53}$.

Para que ações de tratamento e promoção da saúde sejam efetivas deve ser dado destaque, portanto, à capacitação profissional, pois a estrutura imprópria, a intensa rotatividade e o comprometimento heterogêneo dos profissionais com o atendimento impede que se inicie e dê seguimento aos tratamentos em meio às demais funções que estes profissionais exercem ${ }^{41,70}$. Neste cenário, a realização de ações intersetoriais na APS pode aumentar o sucesso de intervenções clínicas de cessação do hábito em pacientes tabagistas, satisfazendo tanto paciente quanto equipe multidisciplinar $^{70}$.

A revisão de literatura realizada permitiu que fossem atingidos os objetivos propostos para esse estudo, gerando a possibilidade de se incrementar a prática de produção de cuidado na APS por meio da adoção de novas estratégias de enfrentamento ao tabagismo ao identificar a recorrência da abordagem individual breve ou intensa a partir do método dos 5A’s (Modelo Transteórico) nos estudos atuais. Os fármacos adesivos de Nicotina e Bupropiona também se mostraram prevalecentes e o uso crescente de tecnologia dura carece ainda de novos estudos que averiguem os seus impactos no tratamento a tabagistas.

Com relação à prática clínica, verificou-se que novos caminhos se abrem para estratégias que, combinadas, podem ser de baixo custo e alta efetividade desde que o corpo de profissionais da APS as conheça e possua acesso às comprovações científicas de sua eficácia para executá-las.

\section{Colaboradores}

MDV Santos, SV Santos e MCGG Caccia-Bava participaram diretamente no planejamento, execução ou análises deste estudo. Todos os autores deste artigo leram e aprovaram a versão final enviada. 


\section{Referências}

1. Associação Médica Brasileira (AMB). Projeto Diretrizes: evidências científicas sobre tabagismo para subsídio ao Poder Judiciário [na internet]. 2013 [acessado 2016 Fev 15]; [71 p.]. Disponível em: http://www.projetodiretrizes.org.br/diretrizes12/tabagismojudiciario.pdf

2. Bully P, Sanchez A, Zabaleta-del-Olmo E, Pombo H, Grandes G. Evidence from interventions based on theoretical models for lifestyle modification (physical activity, diet, Alcohol and tobacco use) in primary Care settings: A systematic review. Prev Med 2015; 76(Supl.):S76-S93.

3. Ramos LR, Malta DC, Gomes GAO, Bracco MM, Florindo AA, Mielke GI, Parra DC, Lobelo F, Simões EJ, Hallal PC. Prevalence of Health promotion programs in primary Health Care units in Brazil. Rev Saúde Públ 2014; 48(5):837-44.

4. Organização Mundial da Saúde (OMS). Report on the global tobacco epidemic. [documento na internet]. 2013. [acessado 2016 Fev 15]; [4 p.]. Disponível em: http:// www.who.int/tobacco/global_report/2013/en/.

5. Valdivieso-López E, Flores-Mateo G, Molina-Gómez JD, Rey-Reñones C, Uriarte MLB, Duch J, Valverde A. Efficacy of a mobile application for smoking cessation in young people: study protocol for a clustered, randomized trial. BMC Public Health 2013; 13:704.

6. Brasil. Ministério da Saúde (MS). VIGITEL Brasil 2014. [documento na internet]. 2014. [acessado $2016 \mathrm{Fev}$ 15]; [154 p.]. Disponível em: http://bvsms.saude.gov. br/bvs/ publicacoes/vigitel_brasil_2014.pdf.

7. Rodrigues NC, Neves RD, Mendes DS, Mendes CP, Martins IH, Reis IN, Lino VT, O’Dwyer G, Daumas RP, Esteves TM, Andrade MK, Monteiro DL, Barros MB. Profile of Brazilian smokers in the National Program for Tobacco Control. Rev Bras Psiq 2015; 37(2):150154.

8. Ripoll J, Girauta H, Ramos M, Medina-Bombardó D, Pastor A, Alvarez-Ossorio C, Gorreto L, Esteva M, García E, Uréndez A, Buades A, Torres E. Clinical trial on the efficacy of exhaled carbon monoxide measurement in smoking cessation in primary Health Care. BMC Public Health 2012; 12.

9. Land TG, Rigotti NA, Levy DE, Schilling T, Warner D, Li W. The effect of systematic clinical interventions with cigarette smokers on quit status and the rates of smoking-related primary Care office visits. PLoS One 2012; 7(7):e41649.

10. Ministério da Saúde (BR). Portaria no 571 de 05 de abril de 2013. Atualiza as diretrizes de cuidado à pessoa tabagista no âmbito da Rede de Atenção à Saúde das Pessoas com Doenças Crônicas do Sistema Único de Saúde (SUS). Diário Oficial da União 2013; 8 abr.

11. Ganong LH. Integrative reviews of nursing research. Res Nurs Health 1987; 10(1):1-11.

12. Whittemore R, Knafl K. The integrative review: updated methodology. J Adv Nurs 2005; 52(5):546-553.

13. Douglas L, Szatkowski L. Socioeconomic variations in access to smoking cessation interventions in UK primary Care: insights using the Mosaic classification in a large dataset of primary Care records. BMC Public Health 2013; 13:546.

14. Almeida GBS, Rodrigues JR, Freire MR. Acessibilidade dos usuários ao Programa de Controle do Tabagismo. HU Revista 2013; 39(3-4):45-50.
15. Agência Nacional de Vigilância Sanitária (ANVISA). Boletim brasileiro de avaliação em tecnologia em saúde 2011 dez.; 17.

16. Rodriguez-Alvarez $\mathrm{M}$, Torán-Monserrat $\mathrm{P}$, Muñoz -Ortiz L, Negrete-Palma A, Montero-Alia JJ, JiménezGonzález M, Zurilla-Leonarte E, Marina-Ortega V, Olle-Borque M, Valentin-Moya E, Cortada-Cabrera A, Tena-Domingo A, Martínez-González S, Vila-Palau V, Ramos-Ordoñez A, Rotllant-Estelrich G, Forcada-Vega C, Borrell-Thió E. Effectiveness of regular reporting of spirometric results combined with a smoking cessation advice by a primary Care Physician on smoking quit rate in adult smokers: a randomized controlled trial ESPIROTAB study. BMC Fam Pract 2011; 12:61.

17. Smith AJB, Tennison I, Roberts I, Cairns J, Free C. The carbon footprint of behavioural support services for smoking cessation. Tob Contr 2013; 22(5):302-307.

18. Irizar-Aramburu MI, Martinez-Eizaguirre JM, Pacheco-Bravo P, Diaz-Atienza M, Aguirre-Arratibel I, PeñaPeña MI, Alba-Latorre M, Galparsoro-Goikoetxea M. Effectiveness of spirometry as a motivational tool for smoking cessation: a clinical trial, the ESPIMOAT study. BMC Fam Pract 2013; 14:185.

19. Martin-Lujan F, Pinol-Moreso JL, Martin-Vergara N, Basora-Gallisa J, Pascual-Palacios I, Sagarra-Alamo R, Aparicio Llopis E, Basora-Gallisa MT, Pedret-Llaberia R, ESPITAP Group. Effectiveness of a structured motivational intervention including smoking cessation advice and spirometry information in the primary Care setting: the ESPITAP study. BMC Public Health 2011; 11:859.

20. Dawson AP, Cargo M, Stewart H, Chong A, Daniel M. Aboriginal Health Workers experience multilevel barriers to quitting smoking: a qualitative study. Int $J$ equity Health. 2012; 11:27.

21. Szatkowski L, McNeill A, Lewis S, Coleman T. A comparison of patient recall of smoking cessation advice with advice recorded in electronic medical records. BMC Public Health 2011; 11:291,

22. Silveira JAA, Resende HMPD, Lucena Filho AMD, Pereira JG. Características da assistência à saúde a pessoas com Diabetes mellitus acompanhadas na Unidade de Saúde da Família Pedregal II, em Cuiabá, MT: reflexões para a equipe de saúde. Mundo Saúde 2010; 34(1):4349.

23. Bass F, Naish B. Advancing preventive Care with clinical tobacco intervention. Can Fam Physician 2014; 60(3):221-222.

24. Codern-Bové N, Pujol-Ribera E, Pla M, González-Bonilla J, Granollers S, Ballvé JL, Fanlo G, Cabezas C, ISTAPS Group. Motivational interviewing interactions and the primary Health Care challenges presented by smokers with low motivation to stop smoking: a conversation analysis. BMC Public Health 2014; 14:1225.

25. Asfar T, Al-Ali R, Ward KD, Vander Weg MW, Maziak W. Are primary Health Care providers prepared to implement an anti-smoking program in Syria? Patient Educ Couns 2011; 85(2):201-205.

26. Mehring M, Haag M, Linde K, Wagenpfeil S, Schneider A. Effects of a guided web-based smoking cessation program with telephone counseling: a cluster randomized controlled trial. J Med Internet Res 2014; 16(9):e218. 
27. Gillaspy SR, Leffingwell T, Mignogna M, Mignogna J, Bright B, Fedele D. Testing of a web-based program to facilitate parental smoking cessation readiness in primary Care. J Prim Care Community Health 2013; $4(1): 2-7$.

28. Mahoney MC, Erwin DO, Widman C, Masucci Twarozek A, Saad-Harfouche FG, Underwood W 3rd, Fox CH. Formative evaluation of a practice-based smoking cessation program for diverse populations. Health educ behav 2014; 41(2):186-196.

29. Karatay G, Kublay G, Emiroglu ON. Effect of motivational interviewing on smoking cessation in pregnant women. J Adv Nurs 2010; 66(6):1328-1337.

30. Shershneva M, Kim JH, Kear C, Heyden R, Heyden N, Lee J, Mitchell S. Motivational interviewing workshop in a virtual world: learning as avatars. Fam Med 2014, 46(4):251-258.

31. Cabezas C, Advani M, Puente D, Rodriguez-Blanco T, Martin C. Effectiveness of a stepped primary Care smoking cessation intervention: cluster randomized clinical trial (ISTAPS study). Addiction 2011; 106(9):1696-1706.

32. Prochaska JO, Butterworth S, Redding CA, Burden V, Perrin N, Leo M, Flaherty-Robb MSN, Prochaska JM. Initial efficacy of MI, TTM tailoring, and HRI's in multiple behaviors for employee Health promotion. Prev Med 2008; 46(3):226-231.

33. Daoud N, Hayek S, Muhammad AS, Abu-Saad K, Osman A, Thrasher JF, Kalter-Leibovici O. Stages of change of the readiness to quit smoking among a random sample of minority Arab - male smokers in Israel. BMC Public Health 2015; 15:672.

34. Perez-Tortosa S, Roig L, Manresa JM, Martin-Cantera $\mathrm{C}$, Puigdomènech $\mathrm{E}$, Roura $\mathrm{P}$, Armengol $\mathrm{A}$, Advani M, ITADI Group. Continued smoking abstinence in diabetic patients in primary Care: A cluster randomized controlled multicenter study. Diabetes Res 2015; 107(1):94-103.

35. Panda R, Persai D, Venkatesan S. Missed opportunities for brief intervention in tobacco control in primary Care: patients' perspectives from primary Health Care settings in India. BMC Health Serv Res 2015; 15:1.

36. Williams RJ, Masica AL, McBurnie MA, Solberg LI, Bailey SR, Hazlehurst B, Kutz SE, Williams AE, Puro JE, Stevens VJ. Documentation of the 5 as for smoking cessation by PCPs across distinct Health systems. Am J Manag Care 2014; 20(3):e35-42.

37. Papadakis S, McDonald P, Mullen KA, Reid R, Skulsky K, Pipe A. Strategies to increase the delivery of smoking cessation treatments in primary Care settings: A systematic review and meta-analysis. Prev Med 2010; 51(3-4):199-213.

38. Varona-Pérez P, Torres-Barbie P, Elejalde-Larinaga AR, Hernández-Caballero EA, Neninger-Vinageras E, Grupo Especial de Trabajo para la prevención y manejo del cáncer de pulmón. Modelo para la prevención y manejo del cáncer de pulmón en Cuba. Rev Cubana Hig Epidemiol 2010; 50(1):37-47.

39. Bonevski B, Paul C, D’Este C, Sanson-Fisher R, West R, Girgis A, Siahpush M, Carter R. RCT of a client-centred, caseworker-delivered smoking cessation intervention for a socially disadvantaged population. $B M C$ Public Health 2011; 11(1):70.
40. Sheffer CE, Barone C, Anders ME. Training nurses in the treatment of tobacco use and dependence: pre- and post-training results. J Adv Nurs 2011; 67(1):176-183.

41. Bass F, Naish B, Buwembo I. Front-office staff can improve clinical tobacco intervention: Health coordinator pilot project. Can Fam Physician 2013; 59(11):e499506.

42. Pengpid S, Peltzer K, Puckpinyo A, Viripiromgool S, Thamma-Aphiphol K, Suthisukhon K, Dumee D, Kngtapan T. Screening and concurrent brief intervention of conjoint hazardous or harmful Alcohol and tobacco use in hospital out-patients in Thailand: a randomized controlled trial. Subst Abuse Treat Prev Policy 2015; 10:22.

43. Marley JV, Atkinson D, Kitaura T, Nelson C, Gray D, Metcalf S, Maguire GP. The Be Our Ally Beat Smoking (BOABS) study, a randomised controlled trial of an intensive smoking cessation intervention in a remote aboriginal Australian Health Care setting. BMC Public Health 2014; 14:32.

44. Díaz-Gete L, Puigdomànech E, Briones EM, Fàbregas-Escurriola M, Fernandez S, del Val JL, Ballvé JL, Casajuana M, Sánchez-Fondevilla J, Clemente L, Castaño C, Martín-Cantera C, Grupo TABATIC. Effectiveness of an intensive E-mail based intervention in smoking cessation (TABATIC study):study protocol for a randomized controlled trial. BMC Public Health 2013; 13:364.

45. Rice VH, Hartmann-Boyce J, Stead LF. Nursing interventions for smoking cessation. Cochrane Database Syst Rev 2013; (8):CD001188.

46. Efraimsson EO, Fossum B, Ehrenberg A, Larsson K, Klang B. Use of motivational interviewing in smoking cessation at nurse-led chronic obstructive pulmonary disease clinics. J Adv Nurs 2012; 68(4):767-782.

47. Castanal-Canto X, Martin-Miguel MV, Herves-Beloso C, Perez-Cachafeiro S, Espinosa-Arevalo MM, Delgado-Martin JL. Working with our smoker patients in primary Care. Analysis of cost-effectiveness. Aten Primaria 2012; 44(11):659-666.

48. Moyer VA. Primary Care interventions to prevent tobacco use in children and adolescents: U.S. preventive services task force recommendation statement. Pediatrics 2013; 132(3):560-565.

49. Patnode CD, O'Connor E, Whitlock EP, Perdue LA, Soh C, Hollis J. Primary Care-relevant interventions for tobacco use prevention and cessation in children and adolescents: a systematic evidence review for the U.S. Preventive Services Task Force. Ann Intern Med 2013; 158(4):253-260.

50. Halladay J, Gianforcaro R. Engaging a network of primary Care practices in an effort to better assist patients in quitting tobacco use. N C Med J 2013; 74(5):402-403.

51. Haas JS, Linder JA, Park ER, Gonzalez I, Rigotti NA, Klinger EV, Kontos EZ, Zaslavky AM, Brawarsky P, Marinacci LX, St Hubert S, Fleegler EW, Williams DR. Proactive tobacco cessation outreach to smokers of low socioeconomic status: a randomized clinical trial. JAMA Intern Med 2015; 175(2):218-226. 
52. Fellows JL, Mularski R, Waiwaiole L, Funkhouser K, Mitchell J, Arnold K, Luke S. Health and economic effects from linking bedside and outpatient tobacco cessation services for hospitalized smokers in two large hospitals: study protocol for a randomized controlled trial. Trials 2012; 13:129.

53. Marley JV, Kitaura T, Atkinson D, Metcalf S, Maguire GP, Gray D. Clinical trials in a remote Aboriginal setting: lessons from the BOABS smoking cessation study. BMC Public Health 2014; 14:579.

54. Huang Y, Lewis S, Britton J. Use of varenicline for smoking cessation treatment in UK primary Care: an association rule mining analysis. BMC Public Health 2014; 14:1024.

55. Nahvi S, Wu B, Richter KP, Bernstein SL, Arnsten JH. Low incidence of adverse events following varenicline initiation among opioid dependent smokers with comorbid psychiatric illness. Drug Alcohol Depend 2013; 132(1-2):47-52.

56. Andreas S, Chenot JF, Diebold R, Peachey S, Mann K. Effectiveness of varenicline as an aid to smoking cessation in primary Care: an observational study. Eur Addict Res 2013; 19(1):47-54.

57. Liberman JN, Lichtenfeld MJ, Galaznik A, Mastey V, Harnett J, Zou KH, Leader JB, Kirchner HL. Adherence to varenicline and associated smoking cessation in a Community-based patient setting. J Manag Care Pharm 2013; 19(2):125-131.

58. Brose LS, West R, McDermott MS, Fidler JA, Croghan E, McEwen A. What makes for an effective stop-smoking service? Thorax 2011; 66(10):924-926.

59. Sicras-Mainar A, Navarro-Artieda R, Diaz-Cerezo S, Marti-Sanchez B, Sanz-De Burgoa V. Abstinence rates with varenicline compared to bupropion and nicotine replacement therapy for quitting smoking in primary Care. Aten Primaria 2011; 43(9):482-489.

60. Cahill K, Stead LF, Lancaster T. Nicotine receptor partial agonists for smoking cessation. Cochrane Database Syst Rev 2010; (12):Cd006103.

61. Omole OB, Ayo-Yusuf OA, Ngobale KNW. Implementing tobacco dependence treatment during clinical consultations: a qualitative study of clinician's experiences, perceptions and behaviours in a South African primary Health Care setting. BMC Fam Pract 2014; 15:85.

62. Parker WA, Steyn NP, Levitt NS, Lombard CJ. They think they know but do they? Misalignment of perceptions of lifestyle modification knowledge among Health professionals. Public Health Nutr 2011; 14(8):1429-1438.

63. Cox LS, Cupertino AP, Tercyak KP. Interest in participating in smoking cessation treatment among Latino primary Care patients. J Clin Psychol Med Settings 2011; 18(4):392-399.

64. Omole OB, Ngobale KNW, Ayo-Yusuf OA. Missed opportunities for tobacco use screening and brief cessation advice in South African primary Health Care: a cross-sectional study. BMC Fam Pract 2010; 11:94.

65. Reisdorfer E, Gherardi-Donato ECdS, Moretti-Pires RO. Significados atribuídos ao uso de álcool e tabaco por profissionais de saúde. Rev Gaúcha Enferm 2013; 34(4):153-160.
66. Sarna L, Bialous SA, Sinha K, Yang Q, Wewers ME. Are Health Care providers still smoking? Data from the 2003 and 2006/2007 Tobacco Use Supplement-Current Population Surveys. Nicotine Tob Res 2010; 12(11):1167-1171.

67. Raupach T, Al-Harbi G, McNeill A, Bobak A, McEwen A. Smoking cessation education and training in U.K. medical schools: a national survey. Nicotine Tob Res 2015; 17(3):372-375.

68. Olano-Espinosa E, Matilla-Pardo B, Minue C, Anton E, Gomez-Gascon T, Ayesta FJ. Effectiveness of a Health Professional Training Program for Treatment of Tobacco Addiction. Nicotine Tob Res 2013; 15(10):1682-1689.

69. Lawn S, Hersh D, Ward PR, Tsourtos G, Muller R, Winefield A, Coveney J. 'I just saw it as something that would pull you down, rather than lift you up': resilience in never-smokers with mental illness. Health Educ Res 2011; 26(1):26-38.

70. Portes LH, Campos EMS, Teixeira MTB, Caetano R, Ribeiro LC. Actions geared to tobacco control: a review of their implementation in Primary Health Care. Cien Saude Colet 2014; 19(2):439-448.

71. Kruse GR, Kelley JH, Linder JA, Park ER, Rigotti NA. Implementation of an electronic Health record-based Care management system to improve tobacco treatment. J Gen Intern Med 2012; 27(12):1690-1696.

72. Boyle RG, Solberg LI, Fiore MC. Electronic medical records to increase the clinical treatment of tobacco dependence: a systematic review. Am J Prev Med 2010; 39(6 Supl. 1):S77-82.

73. Sheffer CE, Anders M, Brackman SL, Steinberg MB, Barone C. Tobacco intervention practices of primary Care Physicians treating lower socioeconomic status patients. Am J Med Sci 2012; 343(5):388-396.

74. Ribeiro LCM, Peixoto MKAV, Weirich CF, Ribeiro JP, Marinho TA. Ações de educação em saúde no combate ao tabagismo: relato de experiência. Ciênc Cuid Saúde 2011; 10(2):345-352.

Artigo apresentado em 22/03/2016

Aprovado em 21/03/2017

Versão final apresentada em 23/03/2017 